\title{
CAPS Mutations Are Potentially Associated with Unexplained Recurrent Pregnancy Loss
}

Hong Pan, ${ }^{* \dagger \ddagger \S}$ Huifen Xiang, ${ }^{* \dagger}$ Jing Wang, ${ }^{\llbracket}$ Zhaolian Wei, ${ }^{* \dagger}$ Yiran Zhou, ${ }^{* \dagger}$ Beihong Liu, ${ }^{\ddagger \S}$ Tengyan Li, ${ }^{\ddagger \S}$ Xu Ma, ${ }^{\ddagger \S}$ Yunxia Cao, ${ }^{* \dagger}$ and Binbin Wang ${ }^{\ddagger \S}$

From the Department of Obstetrics and Gynecology*, The First Affiliated Hospital of Anhui Medical University, Hefei; the Anhui Province Key Laboratory of Reproductive Health and Genetics, ${ }^{\dagger}$ Hefei; the Graduate School of Peking Union Medical College, ${ }^{\ddagger}$ Beijing; the Center for Genetics, ${ }^{\S}$ National Research Institute for Family Planning, Beijing; and the Department of Medical Genetics and Developmental Biology, "School of Basic Medical Sciences, Capital Medical University, Beijing, China

Accepted for publication September 10, 2018.

Address correspondence to Yunxia Cao, Ph.D., Center of Reproductive Medicine, The First Affiliated Hospital of Anhui Medical University, 81 Meishan Rd., Hefei, Anhui 230022, China; or Xu Ma, M.D., National Research Institute for Family Planning, Dahuisi Road No.12, Haidian District, Beijing 100081, China. E-mail: caoyunxia6@ 126.com or maxu@nrifp.org.cn.

\begin{abstract}
Recurrent pregnancy loss (RPL) is a major concern for women's reproductive health. Several studies have proved that genetics is a major factor leading to unexplained RPL, but the maternal pathogenic genes involved in RPL remain largely unknown. A consanguineous family, including the parents who were cousins and their three daughters who had been diagnosed as having nonsyndromic unexplained RPL, was recruited in this study. A rare homozygous variant in calcyphosine (CAPS; ENST00000588776: c.377delC, p.Leu127Trpfs) might be the potential candidate variant for this RPL family through wholeexome sequencing. Sanger sequencing confirmed that the three affected sisters carried the homozygous p.Leu127Trpfs, whereas their parents carried the heterozygous p.Leu127Trpfs. CAPS encodes a $\mathrm{Ca}^{2+}$ binding protein and may play a role in the regulation of $\mathrm{Ca}^{2+}$ transport. Although the precise underlying mechanisms remain unclear, the previous study suggested that they may be involved in cross talk between $\mathrm{Ca}^{2+}$ signaling and CAMP-protein kinase A pathways, which are crucial to embryo implantation and pregnancy maintenance. Knockdown of CAPS expression might promote the expression of secreted phosphoprotein 1 and matrix metalloproteinase 9 , and the release of prostaglandin $E_{2}$, which all played important roles in embryo implantation and early pregnancy maintenance. These results indicated that the autosomal recessive homozygous mutation, p.Leu127Trpfs, in CAPS might be a maternal effect causative mutation of RPL pathogenesis. (Am J Pathol 2019, 189: 124-131; https:// doi.org/10.1016/j.ajpath.2018.09.010)
\end{abstract}

Recurrent pregnancy loss (RPL) is a serious reproductive health problem; according to the American Society for Reproductive Medicine, RPL is defined as two or more clinical pregnancy losses that affect $2 \%$ to $5 \%$ of all couples. $^{1,2}$ The causes of RPL are various, including uterine factors, antiphospholipid syndrome, inherited thrombophilias, and endocrine, cytogenetic, environmental, and psychological factors. ${ }^{2}$ However, after testing for all these causes, some women have unidentified causes and are considered as having unexplained RPL (URPL). ${ }^{3}$

Many studies have proved that genetics is a major factor leading to URPL, and many candidate genes have been identified. A recent meta-analysis, including 428 casecontrol studies, identified 21 variants of 13 genes that might contribute to URPL. ${ }^{4}$ However, owing to the large differences in sample collection between the studies, the associations were all moderate with RPL. Recently, wholeexome sequencing (WES) was used to identify novel causative genes in RPL patients. A study using WES identified compound heterozygous mutations in dynein cytoplasmic 2 heavy chain 1 (DYNC2H1; NM_001080463)

\footnotetext{
Supported by the National Key Research and Development Program of China grants 2018YFC1002801 (B.W.) and 2016YFC1000307 (X.M.), the National Natural Science Foundation of China grant 81501232 (H.X.), the Central Public Interest Scientific Institution Basal Research Fund grant 2018GJM03 (H.P.), and the National Infrastructure of Chinese Genetic Resources grant YCZYPT (2017)01-6 (B.W.).

H.P. and H.X. contributed equally to this work.

X.M., Y.C., and B.W. contributed equally to this work as senior authors. Disclosures: None declared.
} 
and arachidonate 15-lipoxygenase (ALOX15; NM_001140) in miscarriages from two RPL families. ${ }^{5}$ Another recent study using WES in 49 independent URPL patients identified 27 coding heterozygous variants in 22 genes that belonged to distinct pathways related to implantation and pregnancy outcomes. ${ }^{6}$ However, owing to miscarriages or the lack of segregation analysis, limited progress has been made in identifying the maternal pathogenic genes for RPL.

Calcyphosine (CAPS; NM_004058) encodes a $\mathrm{Ca}^{2+}$ binding protein, belonging to the EF hand motif family, that may play a role in the regulation of $\mathrm{Ca}^{2+}$ transport. Although the precise mechanism of CAPS remains unclear, the previous study suggested that it may be involved in the cross talk between $\mathrm{Ca}^{2+}$ signaling and cAMP-protein kinase A (PKA) pathways. $^{8}$ Therefore, CAPS might be related to RPL because of the regulation of $\mathrm{Ca}^{2+}$ signaling and cAMP-PKA pathways, which are crucial to embryo implantation and pregnancy maintenance..$^{9,10}$

Herein, a consanguineous family, including the parents who were cousins and all three daughters who had been diagnosed as having nonsyndromic URPL, was recruited. The phenotype of this family suggested a maternal homozygous mutation shared by the three sisters. To identify the likely causal mutation, WES was performed in the three affected sisters for shared rare homozygous variants, and an autosomal recessive frameshift mutation in CAPS was identified. The aim of this study is to identify a potential maternal effect causative mutation of RPL pathogenesis using WES technology in an RPL family.

\section{Materials and Methods}

\section{Study Subjects}

All subjects were recruited from the Center for Reproductive Medicine at The First Affiliated Hospital of Anhui Medical University between 2015 and 2018. According to the American Society for Reproductive Medicine's criteria, the peripheral blood of the three sisters diagnosed as having nonsyndromic URPL and their parents, who were cousins, was collected. In addition, 264 independent RPL patients were included in the study. All clinical information available is shown in Table 1 . The decidual and placental villi tissues from five independent URPL patients (cases) and five deliberate pregnancy termination cases with no history of spontaneous abortions (controls) were also collected. The study was approved by the Institute Ethics Committee, National Research Institute for Family Planning, and written informed consent was obtained from all subjects.

\section{Whole-Exome Sequencing}

Genomic DNA samples from the three sisters were used to perform WES. Exomes were captured with SureSelect Human All Exon V5 Kit (Agilent Technologies, Santa Clara, CA), and DNA sequencing was performed on the
HiSeq 2500 platform (Illumina, San Diego, CA). BWA version 0.7.12 (http://bio-bwa.sourceforge.net; OMICS $00654)^{11}$ was used for mapping. The variants were referenced to hg19 genome (http://genome.ucsc.edu, last accessed May 2, 2018) and annotated using SAMtools version 0.1 .19 (http://samtools.sourceforge.net). ${ }^{12}$ Rare homozygous mutations [missense, nonsense, frameshift, or splice site variants; frequency, $<1 \%$ in 1000 Genomes Project database, Single Nucleotide Polymorphism Database, National Heart, Lung, and Blood Institute Exome Sequencing Project database, Exome Variant Server (ExAC) database, and Genome Aggregation Database r2.0.2 (http://gnomad.broadinstitute.org)] were retained. Then, the genotypes of the candidate variants were confirmed in the family members by Sanger sequencing. All the coding regions of candidate CAPS gene were sequenced in another 264 independent RPL patients. The primer sequences are shown in Table 2. Mutationtaster (http://www.mutationtaster.org, last accessed April 14, 2018), ${ }^{13}$ PolyPhen-2 version 2.2.2 (http://genetics.bwh.harvard.edu/pph2, last accessed April 14, 2018), ${ }^{14}$ and PROVEAN version 1.1.3 (http://provean. jcvi.org/index.php, last accessed August 17, 2018) $)^{15}$ were used to predict the effect of the mutations on protein structure and function. Conservation analysis was performed using Clustal Omega (https://www.ebi.ac.uk/ Tools/msa/clustalo, last accessed August 14, 2018). ${ }^{16}$

\section{Detection of CAPS Expression in Decidual and Placental Villi Tissues}

The mRNA levels of CAPS in decidual and placental villi tissues from the five URPL patients and the five controls were analyzed by real-time RT-PCR (RT-qPCR). Each sample was analyzed in triplicate, and data were analyzed using the $2^{-\triangle \Delta \mathrm{Ct}}$ method. Melting curve analysis and agarose gel electrophoresis confirmed the amplification specificity. Primers were picked from PrimerBank (https://pga.mgh.harvard.edu/primerbank, last accessed April 5, 2018), and the sequences are shown in Table 2.

\section{Cell Culture and Transient Transfection}

The human endometrial epithelial RL95-2 (CRL 1671; ATCC, Manassas, VA) and human placenta choriocarcinoma JAR (HTB 144; ATCC) cell lines were maintained in medium supplemented with $10 \%$ fetal bovine serum, 100 $\mathrm{mg} / \mathrm{mL}$ penicillin, and $100 \mathrm{mg} / \mathrm{mL}$ streptomycin in $5 \% \mathrm{CO}_{2}$ at $37^{\circ} \mathrm{C}$. For transient transfection, the cells were seeded in 6-well culture dishes and transfected with siRNA-CAPS using Lipofectamine RNAiMAX (Invitrogen, Carlsbad, $\mathrm{CA}$ ), according to the manufacturer's protocol. 
Table 1 Clinical Features of Familial and Independent RPL Patients with Mutations in CAPS

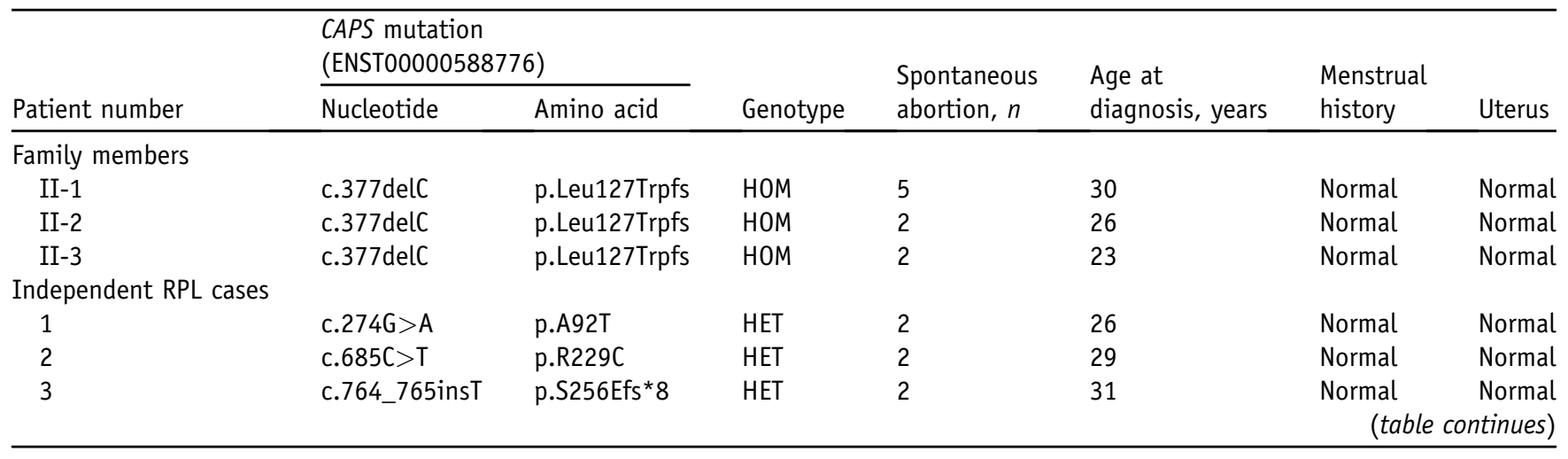

-, negative; ACA, anticardiolipin antibody; ANA, antinuclear antibody; dsDNA, double-stranded DNA; $\beta 2$ GP1, $\beta 2$ glycoprotein 1 ; HET, heterozygous; HOM, homozygous; RPL, recurrent pregnancy loss; TORCH, Toxoplasma gondii others rubella virus cytomegalovirus herpes simplex viruses 1 and 2.

\section{CAPS-Specific SiRNA}

The siRNA set for CAPS, including three pairs of siRNACAPS, positive control, negative control (NC), and FAMlabeled NC, was purchased from Genepharma (Shanghai, China). Real-time RT-PCR and Western blot analysis were used to detect the expression of CAPS. Each assay was run in triplicate with each set of primers, and data were analyzed using the $2^{-\triangle \triangle \mathrm{Ct}}$ method. Melting curve analysis and Sanger sequencing confirmed the amplification specificity. The mRNA level of each sample was normalized to $\beta$-actin mRNA. Densitometry of bands was analyzed using the ImageJ software version k $1.45(\mathrm{NIH}$, Bethesda, MD; https://imagej.nih.gov/ij). The CAPS-homo-919 duplexes were as follows: 5'-GGAUCUGGAGGAGUUCCUUTT- $3^{\prime}$ (forward) and 5'-AAGGAACUCCUCCAGAUCCTT- $3^{\prime}$ (reverse). Cells were seeded in 6-well plates and transfected with CAPS-homo-919 and NC in the following assays.
Detection of Secreted Phosphoprotein 1 and Matrix Metalloproteinase 9 Expression

The human endometrial epithelial RL95-2 cell line was used to detect the biomarkers related to endometrial receptivity and implantation using real-time RT-PCR. Each assay was run in duplicate with each set of primers. The mRNA level of each sample was normalized to $\beta$-actin mRNA. The sequences of the primers for secreted phosphoprotein 1 (SPP1; NM_001251830) and matrix metalloproteinase-9 (MMP9; NM_004994) are listed in Table 2.

\section{Prostaglandin $\mathrm{E}_{2}$ Measurement}

The human endometrial epithelial RL95-2 cell line was used to detect the content of prostaglandin $\mathrm{E}_{2}\left(\mathrm{PGE}_{2}\right)$ release. After treatment, cell-free supernatant was collected and $\mathrm{PGE}_{2}$ content was measured using a $\mathrm{PGE}_{2}$ enzyme-linked

Table 2 Primers for Sanger Sequencing and Real-Time RT-PCR

\begin{tabular}{|c|c|c|c|}
\hline Variable & Identification & Sequences & Length, bp \\
\hline \multirow[t]{5}{*}{ Sanger sequencing } & CAPS-1F & 5'-GAGCCAGCCCACTAAGTCACGT-3' & 903 \\
\hline & CAPS-1R & 5'-CTGTGGCACTGCATCCCTTCCT-3' & \\
\hline & CAPS-2R & 5'-CTCCGCATCTCAGCATCCTCTGT-3' & \\
\hline & CAPS-3F & 5'-GGACTCCTCCCTAAGAGCTGCTGT-3' & 765 \\
\hline & CAPS-3R & 5'-CTGGTCAGCATGGCGTGGGT-3' & \\
\hline \multirow[t]{5}{*}{ Real-time RT-PCR } & $\beta$-Actin-F & $5^{\prime}-\mathrm{CTCCATCCTGGCCTCGCTGT-3^{ \prime }}$ & 117 \\
\hline & & $5^{\prime}$-ACTAAGTCATAGTCCGCCTAGA-3' & \\
\hline & CAPS-qPCR-F & $5^{\prime}$-GTCCAGGGTCACACAGGAATC-3' & 184 \\
\hline & & 5'-GACTGCTTACTCAACTGCCAG- $3^{\prime}$ & \\
\hline & SPP1-qPCR-F & $5^{\prime}-$ CTCCATTGACTCGAACGACTC- $3^{\prime}$ & 230 \\
\hline
\end{tabular}




\begin{tabular}{|c|c|c|c|c|c|c|c|c|}
\hline Hormonal & Karyotype & TORCH & Anti- $\beta 2$ GP1 & $\begin{array}{l}\text { ACA, ANA, and } \\
\text { anti-dsDNA }\end{array}$ & Hepatitis & Infections & Childbearing & Male factor \\
\hline Normal & 46, XX & - & - & - & - & No & 1 & None \\
\hline Normal & 46, XX & - & - & - & - & No & 0 & None \\
\hline Normal & 46, XX & - & - & - & - & No & 1 & None \\
\hline Normal & 46, XX & - & - & - & - & No & 0 & None \\
\hline
\end{tabular}

immunosorbent assay kit, monoclonal (Cayman Chemical, Ann Arbor, MI).

\section{Transwell Migration and Invasion Assays}

Fetal bovine serum - free JAR cells were added to the upper chamber, and $500 \mu \mathrm{L}$ medium with $25 \%$ fetal bovine serum was placed in the lower compartment. After incubation, the nonmigrated cells in the upper chamber were removed with a cotton swab. The migrated cells were fixed with $100 \%$ methanol, stained with $0.5 \%$ crystal violet, and washed with phosphate-buffered saline. The membranes were observed under a light microscope, and three fields were randomly selected to represent each membrane. As for the invasion assay, $70 \mu \mathrm{L}$ Matrigel diluted with Dulbecco's modified Eagle's medium was used to plate each transwell chamber and was incubated for 4 hours at $37^{\circ} \mathrm{C}$, and the same procedure for migration assay was conducted.

\section{Statistical Analysis}

Statistical analysis was performed using GraphPad Prism 5 (GraphPad Inc., San Diego, CA). The independent-sample $t$ test was applied to compare the data, and $P<0.05$ was considered statistically significant.

\section{Results}

\section{Clinical Features of Familial and Independent RPL Patients}

Three sisters (II-1, II-2, and II-3), from a consanguineous Chinese family (Figure 1A), experienced unexplained RPL. Their parents, who were cousins, had given birth to three daughters and one son, without a history of spontaneous miscarriages. The peripheral blood and clinical information from all of them were collected in 2015 (Table 1). The proband (II-1) with the age at diagnosis of 30 years, who had been married for 8 years and had a healthy girl during her second pregnancy, miscarried her first and third to sixth pregnancies for unknown reasons. Her sister (II-2) with the age at diagnosis of 26 years, who had been married for 6 years and had a healthy girl during her second pregnancy, miscarried her first and third pregnancies for unknown reasons. Another sister (II-3) with the age at diagnosis of 23 years, who had been married for 2 years, miscarried twice for unknown reasons. They all had normal karyotype, hormone levels, menstrual history, and uterus. They were negative for TORCH (Toxoplasma gondii others rubella virus cytomegalovirus herpes simplex viruses 1 and 2 ), anti- $\beta 2$ glycoprotein 1, anticardiolipin antibody, antinuclear antibody, anti-
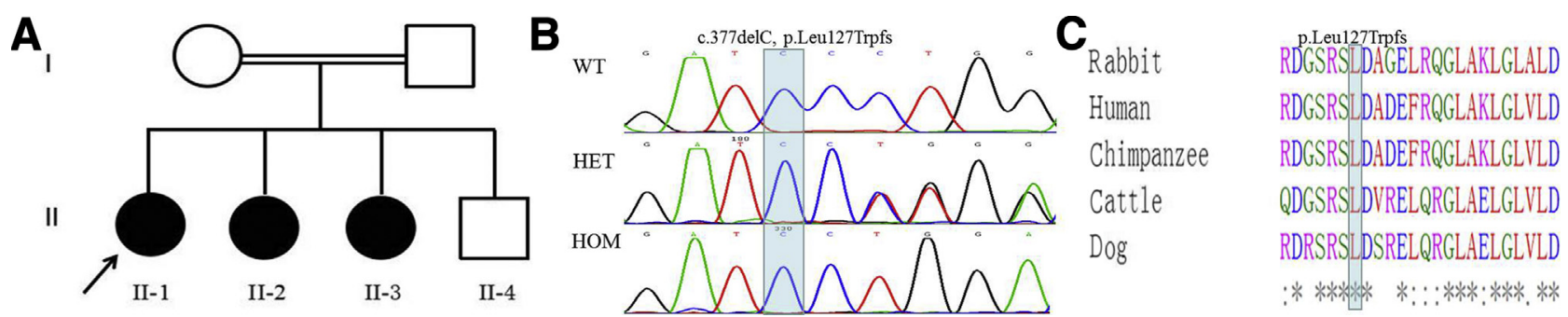

Figure 1 Pedigree of a family with three sisters experiencing unexpected recurrent pregnancy loss (URPL), with homozygous CAPS p.Leu127Trpfs variant. A: The pedigree of the URPL family; the parents were cousins. Whole-exome sequencing was performed on the three affected sisters (II-1, II-2, and II-3). Arrow indicates the proband. B: DNA chromatogram of the CAPS (ENST00000588776: c.377delC, p.Leu127Trpfs) mutation, compared with the wild type (WT); the three sisters carried a homozygous (HOM) variant, and the parents carried a heterozygous (HET) variant. C: This mutation is highly conserved across human, chimpanzee, cattle, rabbit, and dog. Blue boxes indicate the location of the mutated nucleotide base or amino acid residue; asterisks, amino acid residues are the same between different species. 
double-stranded DNA, hepatitis, and infections during screening. Male factor was excluded as well. In addition, the mean age of the 264 independent RPL patients was 29.26 years, and they all experienced at least two spontaneous miscarriages with unknown reasons (Table 1).

\section{Homozygous Frameshift Mutation in CAPS Identified in URPL Family}

In the consanguineous RPL family, three affected sisters (II-1, II-2, and II-3) were chosen for WES. Pedigree analysis suggested the presence of a recessive inheritance model. Therefore, rare homozygous mutations (missense, nonsense, frameshift, or splice site variants; frequency, $<1 \%$ in 1000 Genomes Project database, Single Nucleotide Polymorphism Database, Exome Sequencing Project database, ExAC database, and Genome Aggregation Database) that were shared by all three sisters were retained (Supplemental Table S1). Six nonsynonymous homozygous variants in CAPS (NM_004058), patatin-like phospholipase domain containing 6 (PNPLA6; NM_001166114), semaphorin 6B (SEMA6B; NM_032108), TBC1 domain family member 25 (TBC1D25; NM_002536), WWC family member 3 (WWC3; NM_015691), and zinc finger protein 441 (ZNF441, NM_152355) were identified (Supplemental Table S2 ${ }^{8,17-21}$ ).

Given the frequency and location of the mutations, the effect on protein structure and function predicted by Mutationtaster, PolyPhen-2, PROVEAN, and SIFT, the gene expression profile, the conservation among several species, the published phenotype of knockout mice from Mouse Genome Informatics, the variants reported in the ClinVar database (https://www.ncbi.nlm.nih.gov/clinvar), the function of the genes, and the three sisters' phenotypes, all six rare nonsynonymous homozygous variants were thoroughly screened. The SEMA6B and $W W C 3$ variants were predicted to not alter the protein structure and function and were not conserved among species. The ZNF441 variant was not conserved and had not been previously reported. The TBC1D25 variant was located near the C-terminal end of the TBC1D25 protein, which may not affect the protein features. Furthermore, all these variants have not been reported to relate to human reproductive diseases so far. PNPLA6 was thought to function in the nervous system, and PNPLA6 variants were always related to severe syndromes, such as spastic paraplegia 39 [Online Mendelian Inheritance in Man (OMIM) number 612020], ${ }^{22}$ Boucher-Neuhauser syndrome (OMIM number 215470), ${ }^{17}$ Laurence-Moon syndrome (OMIM number 245800), ${ }^{23}$ Oliver-McFarlane syndrome (OMIM number 275400), ${ }^{23}$ and Gordon Holmes syndrome (OMIM number 212840). ${ }^{24}$ However, the three sisters were healthy and had no sign of any identified disease, except URPL. Furthermore, hypogonadotropic hypogonadism is one of the classic characteristics of Boucher-Neuhauser syndrome and
Gordon Holmes syndrome, which is associated with underdevelopment of the gonads and infertility. ${ }^{17,24}$

Therefore, the homozygous variant in CAPS (ENST00000588776: c.377delC, p.Leu127Trpfs), a frameshift mutation caused by a single-base deletion, was the potential candidate for this RPL family (Figure 1B). The CAPS variant was not previously reported in 1000 Genomes Project, Exome Sequencing Project, and ExAC databases, and was likely to cause a loss-of-function mutation. This amino acid residue is highly conserved across human, chimpanzee, cattle, rabbit, and dog (Figure 1C). Furthermore, no homozygous loss-of-function mutation has been registered in the ExAC database, and the minor allele frequency of any loss-offunction mutation in CAPS is $<0.0001894$, which is low enough to explain the autosomal recessive model of a rare condition. Moreover, CAPS might be involved in both $\mathrm{Ca}^{2+}$ signals and the cAMP-PKA pathway, which are essential for implantation and placental development. ${ }^{9,10}$

Furthermore, Sanger sequencing for all coding exons for CAPS in independent cases with RPL identified three additional heterozygous mutations (ENST00000588776: c.274G >A, p.A92T; c.685C > T, p.R229C; c.764_765insT, p.S256Efs*8) in three patients; however, no homozygous or compound heterozygous variants were found (Table 1). The p.A92T and p.S256Efs*8 variants were not previously reported. The p.R229C variant was reported once in the ExAC database. Among them, a truncated protein would result from the p.S256Efs*8 variant.

\section{Low Expression Levels of CAPS in Decidua and Placental Villi from Independent RPL Patients}

The decidual and placental villi tissues were collected from five independent URPL patients who had experienced two consecutive spontaneous abortions because of unknown reasons. The couples' karyotypes were all normal. Tissues were also collected from five deliberate pregnancy termination cases that had no history of spontaneous abortions as controls. Compared with controls, the expression of CAPS was down-regulated in decidual and placental villous tissues of RPL patients $(P<0.05)$ (Figure 2$)$.

\section{The Most Effective CAPS-Specific siRNA, CAPS- Homo-919}

Real-time RT-PCR and Western blot analysis were used to detect CAPS expression and select the most effective CAPS siRNA. According to the data, CAPS expression in CAPS-homo-919-transfected cells showed a significant decrease compared with the other groups, and the effectiveness of CAPS-homo-919 was best among the three CAPS siRNAs (data not shown). Therefore, CAPS-homo919 was used for CAPS knockdown in the following experiments. 

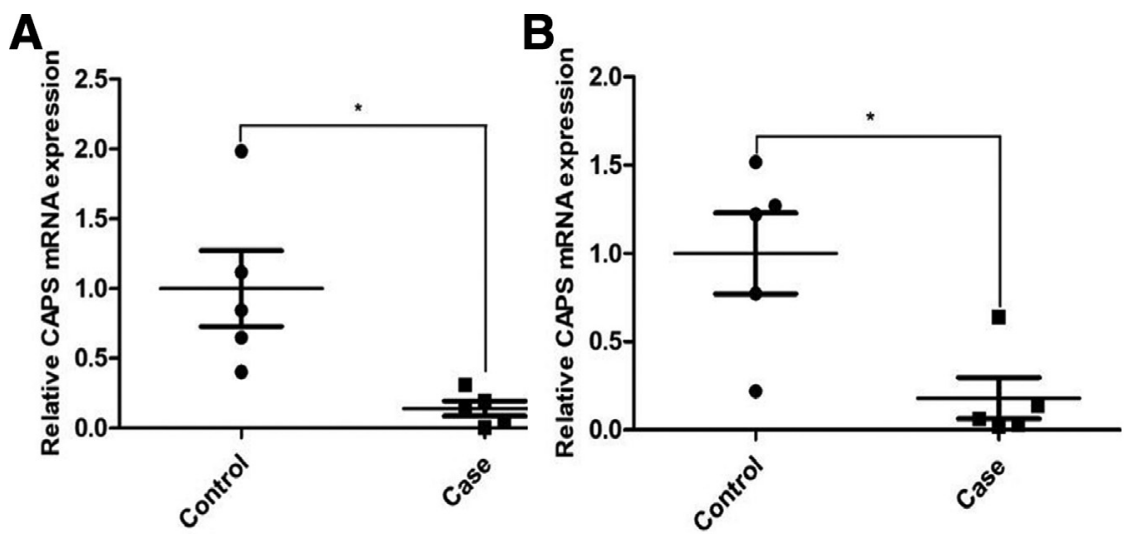

Figure 2 The mRNA levels of CAPS in decidual and placental villi tissues from independent unexpected recurrent pregnancy loss patients. A: Compared with the control, CAPS mRNA expression in decidua is significantly down-regulated in the cases. B: Compared with the control, CAPS mRNA expression in placental villi is significantly downregulated in the cases. ${ }^{*} P<0.05$ ( $t$-test).

\section{Effects of Knockdown CAPS on SPP1 and MMP9 Expression}

To explore the possible role of CAPS in embryo implantation, the expression of several endometrial receptivity markers was detected after CAPS-homo-919 transfection. Compared with controls, Real-time RT-PCR showed that the expression of $S P P 1$ and $M M P 9$ was up-regulated in RL95-2 cells after down-regulation of CAPS expression (Figure 3A). SPPI and MMP9 play important roles in implantation and early pregnancy establishment. ${ }^{25,26}$ The expression levels of $S P P 1$ and $M M P 9$ were up-regulated by 2.04- and 2.82-fold, respectively.

\section{Knockdown CAPS Increases PGE $_{2}$ Release from RL95-2 Cells}

At the epithelial-stromal interface, $\mathrm{PGE}_{2}$, released from endometrial epithelial cells driven by $\mathrm{Ca}^{2+}$ influx, was shown to be essential for stromal cell decidualization and embryo implantation. ${ }^{27}$ The human endometrial epithelial RL95-2 cells are generally used as a model for providing secretory products and receptive epithelial cells. ${ }^{28}$ An enzyme-linked immunosorbent assay showed that CAPS-homo-919 could induce a dramatic increase in $\mathrm{PGE}_{2}$ release from the RL95-2 cells $(P<0.05)$, compared with NC (Figure 3B).

\section{Knockdown CAPS Inhibits JAR Cell Migration and Invasion}

Trophoblast migration and invasion are critical features of implantation and placental development. To examine the possible effect of CAPS knockdown on cell migration and invasion, the transwell assays were performed using JAR cells transfected with CAPS-homo-919 or NC. The transwell assay showed that CAPS-homo-919 could inhibit JAR cell migration compared with $\mathrm{NC}(P<0.01)$ (Supplemental Figure S1A). In addition, the transwell assay with Matrigel revealed that CAPS-homo-919 could affect JAR cell invasion compared with NC $(P<0.01)$ (Supplemental Figure S1B). These results indicated that knockdown of CAPS might lead to a slight decrease in JAR cell migration and invasion.
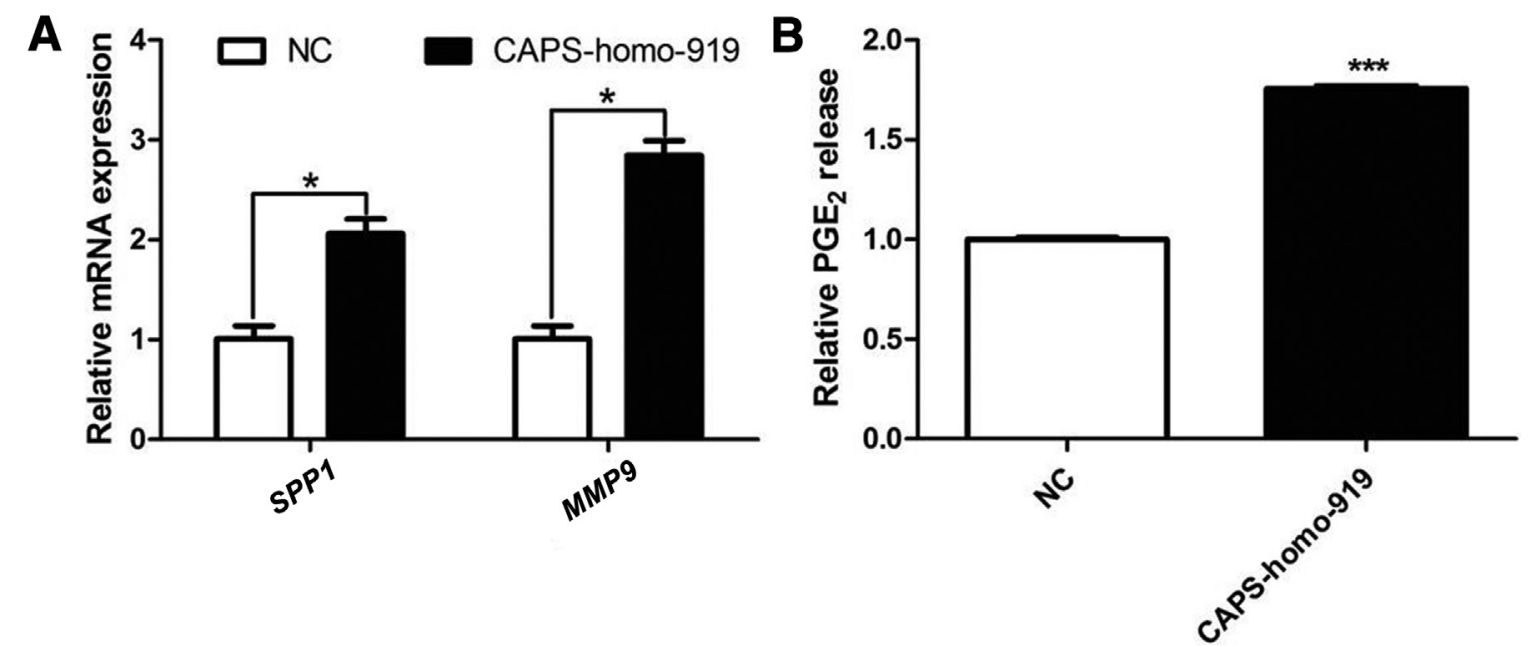

Figure 3 Effects of CAPS knockdown on human endometrial epithelial RL95-2 cell line. A: Knockdown of CAPS expression significantly increases the expression of SPP1 and MMP9, which are factors related to endometrial receptivity. B: Knockdown of CAPS expression significantly promotes prostaglandin $E_{2}$ $\left(\mathrm{PGE}_{2}\right)$ release from the epithelial culture. Data are expressed as means $\pm \mathrm{SEM}(\mathbf{A}$ and $\mathbf{B}) .{ }^{*} P<0.05, * * * P<0.001$ versus NC ( $t$-test). NC, negative control. 


\section{Discussion}

In a consanguineous Chinese family of three sisters with nonsyndromic URPL, an autosomal recessive frameshift mutation was identified in CAPS (ENST00000588776: c.377delC, p.Leu127Trpfs) by WES, which is a possible link to URPL. The results indicated that the homozygous mutation, p.Leu127Trpfs, in CAPS might be the maternal effect causative mutation of RPL pathogenesis.

These data demonstrated that CAPS might be the maternal recessive causative mutation in this nonsyndromic URPL family. Three sisters from a consanguineous Chinese family experienced at least two spontaneous miscarriages for unknown reasons. Pedigree analysis suggested there might be a maternal recessive homozygous mutation shared by the three sisters. Through WES and subsequent bioinformatic analysis, it was found that the homozygous variant in $C A P S$, a frameshift mutation caused by a single-base deletion, was the candidate causative mutation in this RPL family. The CAPS variant (ENST00000588776: c.377delC, p.Leu127Trpfs) had never been reported in 1000 Genomes Project, Exome Sequencing Project, and ExAC databases, and was likely to cause a loss-offunction mutation. Besides, no homozygous loss-of-function mutation has been registered in the ExAC database, and the minor allele frequency of any loss-of-function mutation in $C A P S$ is low enough to explain the autosomal recessive model of this rare condition. To determine the contribution of the $C A P S$ gene to RPL, all the exons of CAPS were screened by Sanger sequencing in 264 independent cases with RPL, and no homozygous or compound heterozygous variants were identified. Three heterozygous mutations in three independent cases with RPL were identified, which might not be responsible for RPL pathogenesis because of heterozygosity because the mother of the three sisters also carried the heterozygous p.Leu127Trpfs and gave birth to four children without a history of RPL. Thus, these data suggested that a biallelic CAPS mutation is a possible link to URPL and that further screening the biallelic CAPS mutation from unrelated families would strengthen the genetic evidence.

The $\mathrm{Ca}^{2+}$ signaling pathway is known to participate in the regulation of several implantation-related events, such as adhesion of the blastocyst to the endometrium, ${ }^{29}$ prostaglandin release from endometrial epithelial cells and the process of stromal cell decidualization, ${ }^{27}$ and the expression of implantation factors. ${ }^{30}$ Similar to the $\mathrm{Ca}^{2+}$ signaling pathway, the cAMP-PKA pathway is also essential for embryo implantation progression. ${ }^{31}$ CAPS encodes a $\mathrm{Ca}^{2+}$-binding protein, which might be involved in cross-signaling between $\mathrm{Ca}^{2+}$ signaling and the cAMP-PKA pathway. ${ }^{8}$ Moreover, real-time RT-PCR results revealed that the expression of CAPS might be downregulated in decidual and placental villous tissues of RPL patients. These data suggested that CAPS might be involved in embryo implantation and early pregnancy maintenance.

The prolonged receptivity state will cause poor embryo implantation and miscarriage. Thus, a proper transient window of endometrial receptivity is essential for embryo implantation. ${ }^{32,33} S P P 1$, expressed by endometrial epithelial cells, was reported to act as an important component of the uteroplacental microenvironment and play an essential role in implantation. ${ }^{25}$ Highly expressed MMP9 can promote the degradation of endometrial extracellular matrix during embryo implantation and may play an important role in the occurrence of RPL. ${ }^{34,35}$ In addition, increased expression of SPPI may increase the expression of MMP $9 .{ }^{36}$ Using the human endometrial epithelial RL95-2 cell line, which is generally used as a model for providing secretory products and receptive epithelial cells, knockdown of CAPS expression was found to increase the expression of SPP1 and $M M P 9$. Besides, the enzyme-linked immunosorbent assay also showed that knockdown of CAPS expression could induce a significant increase in $\mathrm{PGE}_{2}$, an important factor for embryo implantation ${ }^{27}$ and endometrial decidualization, release from the epithelial RL95-2 cells. Therefore, these results indicated that CAPS might be a link to URPL, by regulating the expression of SPPI and MMP9 and the release of $\mathrm{PGE}_{2}$. However, the pathways and mechanisms linking CAPS and RPL require further elucidation.

Besides, to determine the effect of CAPS knockdown on trophoblast cells, a first-trimester trophoblast cell model of JAR choriocarcinoma cells was used. The results confirmed that knockdown of CAPS expression in JAR cells could lead to a slight decrease in cell migration and invasion, which might impair trophoblast infiltration ability. Because the mother of the three sisters gave birth to three daughters carrying homozygous p.L127Wfs*73, the effect of this mutation from the placental side might be limited.

In sum, the results indicated that the autosomal recessive homozygous mutation, p.Leu127Trpfs, in CAPS might be a maternal effect causative mutation of RPL pathogenesis.

\section{Acknowledgment}

We thank the patients and their family members for participating in this study.

\section{Supplemental Data}

Supplemental material for this article can be found at https://doi.org/10.1016/j.ajpath.2018.09.010.

\section{References}

1. Practice Committee of American Society for Reproductive Medicine: Evaluation and treatment of recurrent pregnancy loss: a committee opinion. Fertil Steril 2012, 98:1103-1111

2. El Hachem H, Crepaux V, May-Panloup P, Descamps P, Legendre G, Bouet PE: Recurrent pregnancy loss: current perspectives. Int J Womens Health 2017, 9:331-345

3. Kutteh WH: Novel strategies for the management of recurrent pregnancy loss. Semin Reprod Med 2015, 33:161-168

4. Pereza N, Ostojic S, Kapovic M, Peterlin B: Systematic review and meta-analysis of genetic association studies in idiopathic recurrent spontaneous abortion. Fertil Steril 2017, 107:150-159.e2 
5. Qiao Y, Wen J, Tang F, Martell S, Shomer N, Leung PC, Stephenson MD, Rajcan-Separovic E: Whole exome sequencing in recurrent early pregnancy loss. Mol Hum Reprod 2016, 22:364-372

6. Quintero-Ronderos P, Mercier E, Fukuda M, Gonzalez R, Suarez CF, Patarroyo MA, Vaiman D, Gris JC, Laissue P: Novel genes and mutations in patients affected by recurrent pregnancy loss. PLoS One 2017, 12:e0186149

7. Dong H, Li X, Lou Z, Xu X, Su D, Zhou X, Zhou W, Bartlam M, Rao Z: Crystal-structure and biochemical characterization of recombinant human calcyphosine delineates a novel EF-hand-containing protein family. J Mol Biol 2008, 383:455-464

8. Lefort A, Lecocq R, Libert F, Lamy F, Swillens S, Vassart G, Dumont JE: Cloning and sequencing of a calcium-binding protein regulated by cyclic AMP in the thyroid. EMBO J 1989, 8:111-116

9. Kusama K, Yoshie M, Tamura K, Imakawa K, Isaka K, Tachikawa E: Regulatory action of calcium ion on cyclic AMP-enhanced expression of implantation-related factors in human endometrial cells. PLoS One 2015, 10:e0132017

10. Salker MS, Singh Y, Durairaj R, Yan J, Alauddin M, Zeng N, Steel JH, Zhang S, Nautiyal J, Webster Z, Brucker SY, Wallwiener D, Anne CB, Brosens JJ, Lang F: LEFTY2 inhibits endometrial receptivity by downregulating Orail expression and store-operated $\mathrm{Ca}(2+)$ entry. J Mol Med 2018, 96:173-182

11. Li H, Durbin R: Fast and accurate short read alignment with BurrowsWheeler transform. Bioinformatics 2009, 25:1754-1760

12. Li H, Handsaker B, Wysoker A, Fennell T, Ruan J, Homer N, Marth G, Abecasis G, Durbin R: 1000 Genome project data processing subgroup. The sequence alignment/map (SAM) format and SAMtools. Bioinformatics 2009, 25:2078-2079

13. Schwarz JM, Cooper DN, Schuelke M, Seelow D: MutationTaster2: mutation prediction for the deep-sequencing age. Nat Methods 2014, $11: 361-362$

14. Adzhubei IA, Schmidt S, Peshkin L, Ramensky VE, Gerasimova A, Bork P, Kondrashov AS, Sunyaev SR: A method and server for predicting damaging missense mutations. Nat Methods 2010, 7:248-249

15. Choi Y, Sims GE, Murphy S, Miller JR, Chan AP: Predicting the functional effect of amino acid substitutions and indels. PLOS ONE 2012, 7:e46688

16. Sievers F, Wilm A, Dineen DG, Gibson TJ, Karplus K, Li W, Lopez R, McWilliam H, Remmert M, Söding J, Thompson JD, Higgins DG: Fast, scalable generation of high-quality protein multiple sequence alignments using Clustal Omega. Mol Syst Biol 2011, 7:539

17. Synofzik M, Gonzalez MA, Lourenco CM, Coutelier M, Haack TB, Rebelo A, Hannequin D, Strom TM, Prokisch H, Kernstock C, Durr A, Schols L, Lima-Martinez MM, Farooq A, Schule R, Stevanin G, Marques WJ, Züchner S: PNPLA6 mutations cause BoucherNeuhauser and Gordon Holmes syndromes as part of a broad neurodegenerative spectrum. Brain 2014, 137:69-77

18. Collet P, Domenjoud L, Devignes MD, Murad H, Schohn H, Dauca M: The human semaphorin 6B gene is down regulated by PPARs. Genomics 2004, 83:1141-1150

19. Itoh T, Kanno E, Uemura T, Waguri S, Fukuda M: OATL1, a novel autophagosome-resident Rab33B-GAP, regulates autophagosomal maturation. J Cell Biol 2011, 192:839-853

20. Han Q, Lin X, Zhang X, Jiang G, Zhang Y, Miao Y, Rong X, Zheng X, Han Y, Han X, Wu J, Kremerskothen J, Wang E: WWC3 regulates the Wnt and Hippo pathways via Dishevelled proteins and large tumour suppressor 1, to suppress lung cancer invasion and metastasis. J Pathol 2017, 242:435-447

21. Wang Y, Jiang M, Yao Y, Cai Z: WWC3 inhibits glioma cell proliferation through suppressing the Wnt/beta-catenin signaling pathway. DNA Cell Biol 2018, 37:31-37
22. Rainier S, Bui M, Mark E, Thomas D, Tokarz D, Ming L, Delaney C, Richardson RJ, Albers JW, Matsunami N, Stevens J, Coon H, Leppert M, Fink JK: Neuropathy target esterase gene mutations cause motor neuron disease. Am J Hum Genet 2008, 82:780-785

23. Hufnagel RB, Arno G, Hein ND, Hersheson J, Prasad M, Anderson Y, Krueger LA, Gregory LC, Stoetzel C, Jaworek TJ, Hull S, Li A, Plagnol V, Willen CM, Morgan TM, Prows CA, Hegde RS, Riazuddin S, Grabowski GA, Richardson RJ, Dieterich K, Huang T, Revesz T, Martinez-Barbera JP, Sisk RA, Jefferies C, Houlden H, Dattani MT, Fink JK, Dollfus H, Moore AT, Ahmed ZM: Neuropathy target esterase impairments cause Oliver-McFarlane and LaurenceMoon syndromes. J Med Genet 2015, 52:85-94

24. Topaloglu AK, Lomniczi A, Kretzschmar D, Dissen GA, Kotan LD, McArdle CA, Koc AF, Hamel BC, Guclu M, Papatya ED, Eren E, Mengen E, Gurbuz F, Cook M, Castellano JM, Kekil MB, Mungan NO, Yuksel B, Ojeda SR: Loss-of-function mutations in PNPLA6 encoding neuropathy target esterase underlie pubertal failure and neurological deficits in Gordon Holmes syndrome. J Clin Endocrinol Metab 2014, 99:E2067-E2075

25. Johnson GA, Burghardt RC, Bazer FW, Spencer TE: Osteopontin: roles in implantation and placentation. Biol Reprod 2003, 69: $1458-1471$

26. Vu TH, Werb Z: Matrix metalloproteinases: effectors of development and normal physiology. Genes Dev 2000, 14:2123-2133

27. Ruan YC, Guo JH, Liu X, Zhang R, Tsang LL, Dong JD, Chen H, Yu MK, Jiang X, Zhang XH, Fok KL, Chung YW, Huang H, Zhou WL, Chan HC: Activation of the epithelial $\mathrm{Na}+$ channel triggers prostaglandin $\mathrm{E}(2)$ release and production required for embryo implantation. Nat Med 2012, 18:1112-1117

28. Hannan NJ, Paiva P, Dimitriadis E, Salamonsen LA: Models for study of human embryo implantation: choice of cell lines? Biol Reprod 2010, 82:235-245

29. Thie M, Denker HW: In vitro studies on endometrial adhesiveness for trophoblast: cellular dynamics in uterine epithelial cells. Cells Tissues Organs 2002, 172:237-252

30. Banerjee P, Ghosh S, Dutta M, Subramani E, Khalpada J, Roychoudhury S, Chakravarty B, Chaudhury K: Identification of key contributory factors responsible for vascular dysfunction in idiopathic recurrent spontaneous miscarriage. PLoS One 2013, 8: e80940

31. Kusama K, Yoshie M, Tamura K, Daikoku T, Takarada T, Tachikawa E: Possible roles of the cAMP-mediators EPAC and RAP1 in decidualization of rat uterus. Reproduction 2014, 147: 897-906

32. Salker MS, Nautiyal J, Steel JH, Webster Z, Sucurovic S, Nicou M, Singh Y, Lucas ES, Murakami K, Chan YW, James S, Abdallah Y, Christian M, Croy BA, Mulac-Jericevic B, Quenby S, Brosens JJ: Disordered IL-33/ST2 activation in decidualizing stromal cells prolongs uterine receptivity in women with recurrent pregnancy loss. PLoS One 2012, 7:e52252

33. Achache H, Revel A: Endometrial receptivity markers, the journey to successful embryo implantation. Hum Reprod Update 2006, 12: 731-746

34. Bischof P: Endocrine, paracrine and autocrine regulation of trophoblastic metalloproteinases. Early Pregnancy 2001, 5:30-31

35. Pereza N, Ostojic S, Volk M, Kapovic M, Peterlin B: Matrix metalloproteinases 1,2,3 and 9 functional single-nucleotide polymorphisms in idiopathic recurrent spontaneous abortion. Reprod Biomed Online 2012, 24:567-575

36. Desai B, Rogers MJ, Chellaiah MA: Mechanisms of osteopontin and CD44 as metastatic principles in prostate cancer cells. Mol Cancer 2007, 6:18 